\title{
Improving Measurement Techniques of Power Transformer Insulation: a Study of the Intermolecular Interactions between Water and Vegetable Oil Based Dielectrics
}

\author{
D. Martin ${ }^{1}$, N. Lelekakis ${ }^{2}$, C. Ekanayake ${ }^{1}$, T. Saha ${ }^{1}, \mathrm{H} \mathrm{Ma}^{1}$ \\ ${ }^{1}$ Power and Systems Engineering \\ University of Queensland \\ St. Lucia, QLD 4072 Australia \\ ${ }^{2}$ Centre for Power Transformer Monitoring, Diagnostics and Life Management \\ Monash University \\ Clayton, VIC 3800 Australia
}

\begin{abstract}
The use of water activity probes immersed in the vegetable oil dielectric of a transformer requires a fresh look at the equations, and assumptions, behind the conversion of water activity to concentration of dissolved water. This is important because water negatively affects the effectiveness of insulation.

A large volume of work has been performed on mineral oil based dielectrics to determine mathematical relationships. However, there is a lack of data on whether these relationships hold for vegetable oil. One example is the appropriateness of using the Henry's law coefficient of proportionality to calculate the concentration of water dissolved in the fluid from its water activity. This paper presents research investigating the water activity-concentration relationship for one vegetable oil dielectric. It was found that, at room temperature under the prescribed conditions, using Henry's law is appropriate.
\end{abstract}

\section{INTRODUCTION}

It is important to measure the water content of an oil dielectric for a number of reasons. For instance, water causes the dielectric strength of oil to fall [1]. When considering a mixture of insulation materials, for instance cellulose and oil together, the water content of paper can be inferred from the dryness of the oil and using cellulose adsorption isotherms [2].

Two techniques to measure the water content of oil are those based on a Karl Fischer (KF) chemical titration, relatively common, and using a water actively probe, not so common in the industry. A water activity probe has the advantage that it is an online technique so can be used in remote monitoring of transformers, whereas the KF titration cannot.

Although KF and water activity both measure water, they actually measure different quantities. KF measures the mass of water dissolved in the oil while water activity reflects the partial pressure of dissolved water. It is useful to be able to convert between the two in order to apply the cellulose adsorption isotherms.

A water activity probe measurement can be converted into concentration of dissolved water using (1) and (2) [3], where $\mathrm{C}$ is the concentration of water dissolved in the oil in parts per million (ppm) by mass, $\mathrm{a}_{\mathrm{w}}$ is the water activity measurement which is a decimal fraction with a range of 0 to 1 , and $S_{(T)}$ is the solubility of water in the oil at that temperature (in ppm). In (2) constants $A$ and $B$ are dependent on the type of oil and its age, which must be known, and $T$ is the temperature in kelvin.

$$
\begin{aligned}
& C=a_{W} \times S_{(T)} \\
& \log S_{(T)}=A-\frac{B}{T}
\end{aligned}
$$

Water activity is equal to the partial pressure of water vapor, $p$, divided by the vapor pressure of pure water at that temperature, $p_{0}$, (3) [4]. The approximation of water activity and the ratio of concentration of water dissolved in the oil, $c$, to the solubility of water in the oil at that temperature, $s$, follows on from Henry's law [5], shown in (4). Equation (4) shows that the pressure of a solute's vapor, $p$, is proportional to its concentration dissolved in the solvent $c . k$ is the constant of proportionality (Henry's constant).

$$
\begin{aligned}
& a_{w}=\frac{p}{p_{0}} \approx \frac{c}{s} \\
& p=k \times c
\end{aligned}
$$

Determining the A and B coefficients for use in (2) also assumes that using Henry's law is valid. The general method to ascertain the coefficients is as follows. An oil is heated in steps. On each step the water activity is measured, and the concentration of water is measured using a Karl Fischer titration. The solubility of the oil to water at that temperature is calculated using (1). When the solubility is found at a number of temperatures plotting the temperature against the $\log$ of solubility yields a straight line, where $\mathrm{A}$ is the $\mathrm{y}$ intercept and B is the gradient. If however Henry's law did not apply then the solubility calculation will require modification. 
As far as we are aware the assumption for (1) to (4) have not been tested on vegetable oil, which could result in incorrect water concentrations being determined.

\section{BACKGROUND}

\section{A. Solubility Calculation}

The solubility of a vegetable oil to water has been calculated by Lewand [6] using (2). This equation is derived from (5) being rearranged into $(6)[7,8]$, which shows the relationship between the molar solubility of one fluid in another, $N$, and the change in heat of solution when this mixture forms, $\Delta H . T$ is the temperature in kelvin and $R$ is the gas constant. Solubility $N$ is expressed as a ratio of molar masses, rather than as the mass ratio $S$. The molar mass of a compound is proportional to its actual mass, so $S$ can be substituted for $N$, although the coefficients will differ.

$$
\begin{aligned}
& \Delta H=R T^{2}\left(\frac{d \ln N}{d T}\right) \\
& \ln N=-\frac{\Delta H}{R T}+C
\end{aligned}
$$

When $\Delta \mathrm{H}$ is assumed to be constant across the temperature range of interest (6) can be reduced to (1). The advantage to using this equation is that it is easy to apply to measurement data. The constants $A$ and $B$ can be determined graphically from plotting the linear relationship $\log S$ against 1/T.

However, Mackey notes that it is incorrect to assume that the heat of solution is not affected by temperature [9]. They warn that this equation is only applicable over narrow temperature ranges where the enthalpy of vaporization is relatively constant, and instead recommends using the three coefficient Antoine equation.

$$
\log p=A-\frac{B}{T+C}
$$

A drawback however is that as (7) has three coefficients it can be more time consuming, and difficult, to solve with a minimal data set of measurements.

Tsonopoulos took (6) and assumed that $\Delta H$ is a linear function of temperature, as opposed to it being constant [10]. The rearrangement and integration of (5) now results in (8). Tsonopoulos then applied this equation to alkanes and cyclohexanes finding a good agreement. Alkane and cyclohexane structures are found in transformer mineral oil, although not in vegetable oil.

$$
\ln N=A+\frac{B}{T}+C \ln T
$$

Yaws and his research students investigated the solubility of alkanes (paraffins) and cycloalkanes (naphthenes) using (9), which they based on (8) although with solubility being expressed as mass concentration $S$ rather than molar concentration $N[11$ - 12]. Yaws determined the coefficients $A$, $B$ and $C$ for a large number of isomers, showing them to asymptote towards certain values, depending on both carbon number and type of structure.

$$
\log _{10} S=A+\frac{B}{T}+C \log _{10} T
$$

The difference between (2) and (9) is the correction given by the third term, $C \log _{10} T$. In order to determine the percentage error from using a two coefficient calculation, rather than three, we calculated the solubility of a hydrocarbon with a molar mass similar to those found in naphthenic transformer oil. According to Lipscomb the average carbon number of the molecules constituting mineral oil is 18 [13]. From Yaws' we chose tetradecylcyclopentane, which with a carbon number of 19 and molar mass of $267 \mathrm{~g} / \mathrm{mol}$ is representative. Its solubility to water at $303 \mathrm{~K}$ is close to that of transformer mineral oil, $\approx 60 \mathrm{ppm}$.

A straight line was fitted to the plot, and then the A and B coefficients were determined. Equation (2) was used to calculate solubility. The calculations from both solubility equations are markedly close to one another, with a maximum relative difference of only one percent. Therefore, errors due to the precision of the equipment used to measure water content could be more significant that the error caused by omitting a third constant.

TABLE I

COMPARISON OF SOLUBILITY CALCULATIONS FROM TwO AND THREE COEFFICIENT CALCULATIONS

\begin{tabular}{|c|c|c|c|}
\hline $\begin{array}{c}\text { Temperature } \\
(\text { kelvin) }\end{array}$ & $\begin{array}{c}\text { Solubility using 3 } \\
\text { coefficient equation } \\
(\mathrm{ppm})\end{array}$ & $\begin{array}{c}\text { Solubility using 2 } \\
\text { coefficient equation } \\
(\mathrm{ppm})\end{array}$ & $\begin{array}{c}\text { Difference } \\
(\%)\end{array}$ \\
\hline 303 & 76 & 75 & -1 \\
\hline 313 & 99 & 99 & 0 \\
\hline 323 & 129 & 130 & +1 \\
\hline 333 & 170 & 171 & +1 \\
\hline 343 & 223 & 224 & 0 \\
\hline 353 & 294 & 294 & 0 \\
\hline 363 & 388 & 386 & -1 \\
\hline 373 & 513 & 507 & \\
\hline
\end{tabular}

III. USE OF WATER ACTIVITY TO CALCULATE MASS OF DISSOLVED WATER

Roddy and Coleman showed that water content of benzene, n-hexane and cyclohexane follows Henry's law [5], and so (3) is valid. This result was backed up by $\mathrm{Du}$, who found a straight line relationship between relative humidity, a function of pressure, and dissolved water concentration [14]. However, the range of Du's result becomes larger as the relative humidity increases. Therefore, it could be that although the relationship between relative humidity and dissolved water concentration in not exactly a straight line, however, it is still appropriate for use.

Golovan showed that the Henry's law relationship does not apply to aged oil, citing that the degree of non-linearity is dependent on the concentration of acids dissolved in the oil [15]. No explication was given on the mechanism causing the deviation. The acid number of vegetable oil is higher than that of mineral oil, however, the processes creating acids differ in these types of oil. In mineral oil oxidation reactions create stronger acids such as methanoic (formic) and ethanoic (acetic) acids. However, in vegetable oil the ester link can break and a weaker, fatty acid is detached. 
Oil acidity testing only determines the amount of a base required to neutralize all the acids in a mass of oil. The test does not determine the type or aggressiveness of the acid. Typical values for acid number, according to standard ASTM D974, are <0.01 for mineral oil and 0.02 for FR3, both in terms of $\mathrm{mg} \mathrm{KOH} / \mathrm{g}$ oil. However, in use we have observed FR3 acidity levels up to $0.05 \mathrm{mg} \mathrm{KOH} / \mathrm{g}$ oil.

Of interest is whether the acid content of a vegetable oil will result in the vapor pressure-water concentration deviating from that specified by Henry's law. This could result in a relationship which takes into account the acid value of the vegetable oil

We set out to investigate the applicability of using equations (1) to (4) to model the concentration of water dissolved in vegetable oil. This was done by graphing the concentration of dissolved water, ppm, against its measured water activity at various temperatures.

\section{INVESTIGATION}

The investigation had two aims. The first was to determine whether a Henry's law coefficient of proportionality holds for vegetable oil, which in this case was the Envirotemp FR3 natural ester dielectric. The second was to ascertain whether the acid concentration of the oil causes a deviation from Henry's law, and if it did the degree of impact. The general strategy was to add water in steps, and when dissolution had occurred measure both water activity and dissolved water content (by KF titration).

An auto-pipette was used to add water into the oil, as the oil was continually stirred. The quantity of water added, $0.16 \mathrm{ml}$, was to raise the water activity by 0.1 on each step. The oilwater mixture within the vessel was checked visually for dissolution. If bubbles of water were present their surface will refract light passing through the oil, causing distortions. In practice at least seven hours was given between adding water and sampling. Water was either added in the morning, and the oil sampled in the late afternoon, or the water was added in the late afternoon and then sampling preformed the following morning. For the wettest sample the oil was left to mix for five days, because after seven hours the oil was slightly cloudy, so longer was given to determine whether more time was required for dissolution. Two samples were extracted for KF titration, one other seven hours and the other after five days. The water activity was the same, 0.94 , at both times. The seven-hour sample appeared a slightly darker shade of green than the five-day sample, possibly indicating that the water had not completely dissolved. However, the KF results were very similar to each other.

\section{A. Method}

The equipment was set up in an air conditioned laboratory, which had a steady temperature of between $22{ }^{\circ} \mathrm{C}$ and $23{ }^{\circ} \mathrm{C}$. Having a steady temperature was very important when investigating very high water activity because a slight fall in temperature could cause the system to reach saturation and thus dew to form, when water is precipitated. This could then affect the relationship between water activity and mass of dissolved water.

A three-liter glass vessel was set up with a water activity probe (Vaisala MMT 330 with MMT 337 probe) and a port with valve to draw samples of vegetable oil for KF analysis. The system is shown in Fig.1. The extraction port was set up so the volume of oil sampled was very close to the tip of the water activity probe. The vessel was placed on top of a magnetic stirrer hotplate. Two liters of vegetable oil (Envirotemp FR3) were dried under vacuum and then poured into the vessel. The stirrer was turned on and the water activity probe output left to settle for thirty minutes.

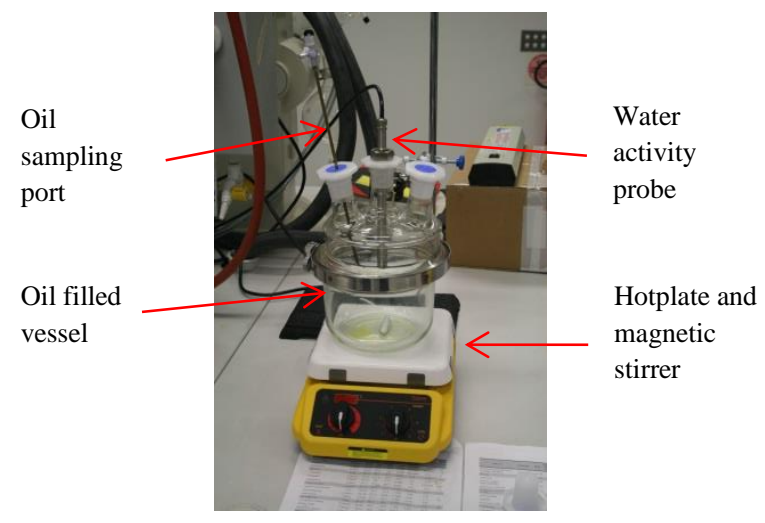

Fig. 1. Experimental setup

Oil was removed for analysis using a $50 \mathrm{ml}$ glass syringe by drawing $30 \mathrm{ml}$ of oil through the sampling valve. The syringe was flushed with oil first, and great care was taken to ensure that any air bubbles were expunged. The sample was also checked visually to ensure the absence of water bubbles (especially after water had been added to ensure proper dissolution).

The KF titration was performed in two laboratories, allowing the measurement results to be checked. Both laboratories used a volumetric measurement technique, where the density of the fluid must be known. The density of soybean is $0.9193 \mathrm{~g} / \mathrm{ml}$ at $23.9{ }^{\circ} \mathrm{C}$ [16], agreeing the $0.92 \mathrm{~g} / \mathrm{ml}$ published by the manufacturer [17]. Obviously, the temperature of the oil in the syringe must be in equilibrium with the measuring lab, i.e. not immediately tested on delivery.

An exact volume of oil was drawn using either a syringe or auto-pipette. The syringe method involved the operator drawing $1.1 \mathrm{ml}$ of vegetable oil, weighing $1 \mathrm{~g}$, from the main glass sampling syringe. The auto-pipette method involved setting the device to extract a given volume of liquid, $0.6 \mathrm{ml}$. We preferred the syringe method because the vegetable oil was too viscous for use with the pipette; it was difficult to ensure that the pipette had correctly deposited the entire sample into the KF titrator. In practice two or three discharges of the auto-pipette were required to fully deposit the sample.

\section{RESULTS}

The relationship between water activity and concentration of dissolved water, for room temperature, is shown in Fig. 2. The 
theoretical dissolved water content, using (1) and (2) with coefficients $\mathrm{A}=6$ and $\mathrm{B}=881.39$ [2], is also given for comparison. The acid number of this oil was low, measured to be only $0.105 \mathrm{mg} \mathrm{KOH} / \mathrm{g}$ oil.

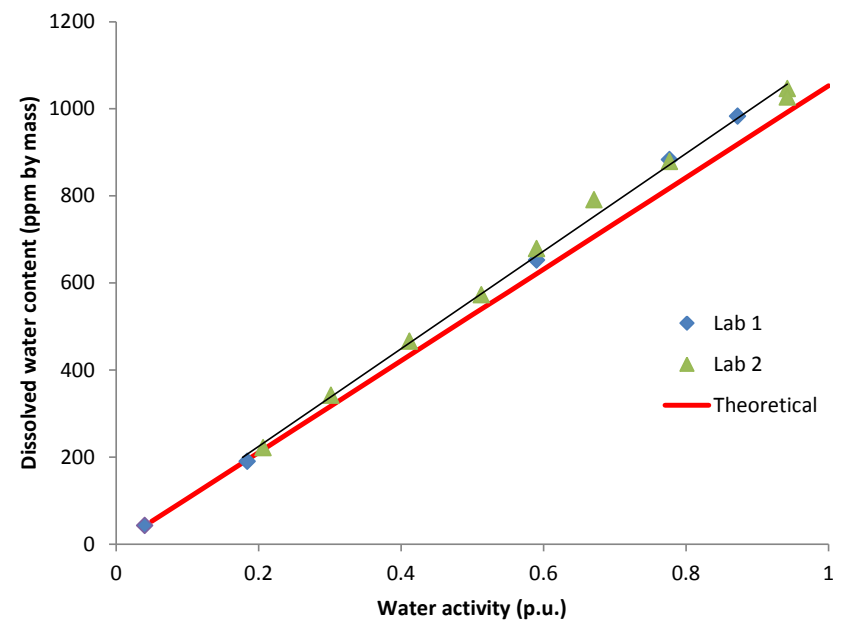

Fig. 2. Plot of water activity and dissolved water content

The correlation between the measured points and theoretical calculated concentration is very close, with the difference appearing insignificant. The A and B coefficients were derived from measurements, thus, there could have been an experimental error in their value. Plotting a linear trend-line along the data and extrapolating to $\mathrm{a}_{\mathrm{w}}=1$ indicates a solubility of $1121 \mathrm{ppm}$ at room temperature, very close to the published value, $0.11 \%$, for soybean oil [18].

Since the measurements are not observed to significantly deviate from a straight line it is concluded that Henry's law of proportionality is being observed. Therefore, using (4) is correct. The deviation from linearity shown by Golovan was not observed. Possible explications include: the type of acid (in particular its dissociation constant) affects any deviation more than its acid number, or, it is not the acid per se which causes a deviation but the general by-products of oxidation. Carboxylic acids are also created during oxidation of oil.

The next steps are to firstly investigate the solubility of vegetable oil to water at a higher temperature, and so check the accuracy of using (2) with the A and B coefficients from [2]. Then secondly investigate effect of different carboxylic and fatty acids, similar to those found in ageing transformer oil, on any deviation from Henry's law.

\section{CONCLUSIONS}

Under room temperature conditions, the vegetable oil-water mixture closely followed the Henry's law of proportionality. Any deviation is therefore too insignificant to notably affect using Henry's law to calculate the dissolved water content, in ppm, from the water activity measurement.

The next steps will be to analyze the behavior of vegetable oil at a higher temperature, as this will check the precision of the A and B solubility coefficients, along with (2), to calculate the ppm of water from water activity. The effect of acids on linearity will also be investigated, and if necessary (2) will be changed to reflect the acid content of oil.

\section{ACKNOWLEDGEMENTS}

The authors wish to thank Cherie Gilbert and Tony Tuong Ngo of Oil Testing Services, Powerlink Queensland, for their technical expertise and input, and Grant Edwards, Australian Institute for Bioengineering and Nanotechnology, for helping set up the laboratory equipment.

The authors also express their gratitude to Lachlan Pill, student, for his assistance helping produce the measurements.

\section{REFERENCES}

[1] D. Martin and Z. D. Wang, "A Comparative Study of the Impact of Moisture on the Dielectric Capability of Esters for Large Power Transformers", IEEE Annual Report Conf. on Elect. Insul. and Dielectric Phenomena, pp. 409 - 412, USA, 2006.

[2] D. Martin, C. P. Perkasa, and N. Lelekakis, "Measuring Paper Water Content of Transformers: A New Approach Using Cellulose Isotherms in Non-Equilibrium Conditions", IEEE Trans. Power Del., in Press.

[3] Vaisala, "HUMICAP Moisture and Temperature Transmitter Series for Oil MMT 310", Finland, 2008.

[4] Dictionary of Food Science and Technology (2nd Edition), International Food Information Service (IFIS Publishing), International Food Information Service, 2009.

[5] J. W. Roddy and C. F. Coleman, "Solubility of Water in Hydrocarbons as a Function of Water Activity", Talanta, Vol. 15, pp. 1281 - 1286, 1986.

[6] L. Lewand, "Laboratory Evaluation of Several Synthetic and Agricultural-Based Dielectric Liquids", Doble International Client Conference, USA, 2001.

[7] G. B. Howe, M. E. Mullins, and T. N. Rogers, "Evaluation and Prediction of Henry's Law Constants and Aqueous Solubilities for Solvents and Hydrocarbon Fuel Components Vol 1: Technical Discussion", Air Force Engineering and Services Center, Florida, USA, 1987.

[8] R. R. Hibbard and R. L. Schalla, "Solubility of Water in Hydrocarbons", National Advisory Committee for Aeronautics, Washington, USA, 1952.

[9] D. Mackay and W. Y. Shiu, "A Critical Review of Henry's Law Constants for Chemicals of Environmental Interest", J. Phys. Chem., Vol. 10, No. 4, 1981

[10] C. Tsonopoulos, "Thermodynamic Analysis of the Mutual Solubilities of Hydrocarbons and Water", Fluid Phase Equilibria, Vol. 186, pp. 185 $-206,2001$.

[11] C. L. Yaws and P. M. Rane, "How Temp. Affects $\mathrm{H}_{2} \mathrm{O}$ Solubility in Alkanes", Oil \& Gas Journal, $12^{\text {th }}$ June 2010, USA.

[12] C. L. Yaws and U. Yadav, "How Temp. Affects $\mathrm{H}_{2} \mathrm{O}$ Solubility in Cycloalkanes", Oil \& Gas Journal, $1^{\text {st }}$ September 2012, USA.

[13] H. G. Erdman, Electrical Insulating Oils, American Society for Testing and Materials, USA, 1988.

[14] Y. Du, A. V. Mamishev, B. C. Lesieutre, M. Zahn \& S.H. Kang, "Measurements of Moisture Solubility for Differently Conditioned Transformer Oils", IEEE Trans. on Dielectric and Elect. Insul., Vol. 8, No. 5, Oct. 2001.

[15] G. D. Golovan, T. B. Zhilyaev, A. I. Panchenko \& V. S. Krivenkaya, "Water Solubility in Transformer Oils with Various Hydrocarbon Compositions", Chemistry and Technology of Fuels and Oils, No. 8, pp. 18 - 20, Springer New York, 1984.

[16] H. Noureddini, B. C. Tech, and L. David Clements, "Densities of Vegetable Oils and Fatty Acids", Journal of the American Oil Chemists Society, Vol 69, Iss. 12, pp. 1184-1188, USA, 1992.

[17] Cooper Industries, Envirotemp FR3 Fluid testing guide, Cooper Industries Inc, Waukesha, USA, 2004.

[18] E. G. Hammond, Soybean Oil, in Bailey's Industrial Oil and Fat Products $6^{\text {th }}$ ed. John Wiley \& Sons, USA, 2005. 